Article

\title{
Design, Modeling and Control of Magnetic Bearings for a Ring-Type Flywheel Energy Storage System
}

\author{
Chow-Shing Toh and Shyh-Leh Chen * \\ Department of Mechanical Engineering and Advanced Institute of Manufacturing with High-Tech Innovations, \\ Chiayi 62102, Taiwan; mydennistcs@gmail.com \\ * Correspondence: imeslc@ccu.edu.tw; Tel.: +886-5-272-0411 (ext. 33320) \\ Academic Editor: Paul Stewart
}

Received: 19 August 2016; Accepted: 2 December 2016; Published: 14 December 2016

\begin{abstract}
This study is concerned with the magnetic force models of magnetic bearing in a flywheel energy storage system (FESS). The magnetic bearing is of hybrid type, with axial passive magnetic bearing (PMB) and radial hybrid magnetic bearing (HMB). For the PMB, a pair of ring-type Halbach arrays of permanent magnets are arranged vertically to support the rotor weight. For the HMB, a set of ring-type Halbach array is placed on the rotor side, which corresponds to coil sets on the stator side. The HMB can produce both attraction and repulsion forces on the radial direction, depending on the direction of the coil currents. It is found that the ring-type configuration and the differential winding scheme for coil sets can yield linear magnetic force models for both PMB and HMB. Based on the obtained magnetic force model, an integral sliding mode controller is designed for the stable rotor levitation in the radial direction. The experimental results show that the rotor can be stabilized to the bearing center, verifying the accuracy of the magnetic force models and effectiveness of the levitation controller.
\end{abstract}

Keywords: Halbach array; magnetic bearing; flywheel battery; nonlinear control

\section{Introduction}

Energy issues have attracted global attention in recent years, especially since accidents occurred in Fukushima, Japan, in 2011. It is an inevitable trend to adopt renewable energies in building a sustainable, low-carbon energy supply system [1]. With the increasing percentage of renewable energy, the energy storage system plays a key role in the energy supply chain. It can provide balancing to the electricity grid that can yield more flexibility in a smart grid. In particular, it can provide a back-up to intermittent renewable energy sources such as solar power and wind turbine. Energy storage system also plays a significant role in emergency preparedness and in increasing overall grid resilience [2,3].

There exist many different types of energy storage systems. Compared to other energy storage systems, the flywheel energy storage system (FESS) possesses many advantages, including power density, charge/discharge response time, energy recovery efficiency, life cycle cost, and impact to the environment [4]. The FESS stores the rotational energy in a massive rotating rotor (the flywheel). The energy is maintained in the flywheel by keeping it at a constant speed. In the charge mode, the input energy will increase the rotation speed to a higher level. In the discharge mode, electricity is extracted from the system by the power electronics module to the load and the rotation speed will decrease.

The design of the rotor is an important issue for FESS. Most designs adopt the shape of disk-like flywheel that is made of high-strength carbon filaments [5,6]. Recently, a ring-type FESS was proposed [7]. It adopted a thin-walled composite hoop as the rotor. Such design can allow reaching higher rotor tangential speed. In addition, the ring-type rotor possesses larger mass moment of inertia 
for the same rotor mass. Note that rotation energy is proportional to the mass moment of inertia. As a result, the specific energy and energy density of the FESS can be improved.

Note also that for a given rotor, the rotation energy is proportional to the square of the rotor speed. Thus, another way of increasing the specific energy and energy density for the FESS is to increase the maximum allowable rotation speed. In this regard, magnetic bearing is the key component in FESS due to its non-contact nature for achieving high rotation speed. Because of the non-contact nature, it possesses the advantages of no friction, less energy loss, low maintenance costs, long system life, and environmental friendliness [8]. Moreover, the optimization on the bearing can be designed for maximum magnetic force with a given current density $[9,10]$, even the eddy currents induced due to the changing currents will result in considerable magnitude decrease and phase lag of stiffness [11].

In general, there are three types of magnetic bearings: passive, active, and hybrid. Passive magnetic bearing (PMB) has the advantage of no energy consumption, but cannot be used alone for stable levitation. Stable levitation can be achieved by the active magnetic bearing (AMB) that uses the electromagnets to actively control the magnetic forces. However, AMB has the disadvantage of higher cost and energy consumption. Hybrid magnetic bearing (HMB) is an integration of PMB and AMB. In other words, some degree-of-freedoms (DOF) of the rotor are levitated by PMB and the others are levitated by AMB [12].

$\mathrm{AMB}$ is the most popular magnetic bearing for the application in FESS. The common design of AMB uses four independent coils and generates forces in two perpendicular directions. For each direction on $X-Y$ plane, two opposite pairs of electric coils are used. A linearization of the quadratic force-current relationship is achieved by applying differential driving currents with the constant bias current [8]. However, AMBs are open-loop unstable systems, so that a feedback control algorithm, such as the proportional-integral-derivative (PID) controller [13], linear-quadratic-Gaussian control [14], and sliding mode control [15], is required to maintain the stability of the system. In an AMB-rotor system, the unbalance compensation can suppress the rotor vibration and improve the rotary precision. A method, which is based on the seeking unbalance mass position, was proposed for unbalance compensation via AMB [16]. Moreover, the high energy consumption of AMB makes it detrimental to the application of energy system.

In this study, the ring-type FESS proposed in [7] is followed. The FESS contains two sets of axial PMB for the levitation of the rotor weight. In doing so, the energy consumption induced by levitating the heavy rotor weight can be eliminated. The system also contains two sets of radial HMB to actively control the rotor at the bearing center. In other words, the rotor is actively controlled by HMB in the radial directions and passively supported by $\mathrm{PMB}$ in the axial direction [12]. Both the stator and rotor of the axial PMB consist of permanent magnet rings arranged in the Halbach array. On the other hand, while the rotor of the radial HMB is also equipped with permanent magnet rings arranged in the Halbach array, the stator is inserted with eight sets of coils. A Halbach array is a special arrangement of permanent magnets that augments the magnetic field on one side of the array while cancelling the field to near zero on the other side [17]. The advantage of Halbach array arrangement is obvious since the magnetic field used for levitation is maximized. A number of Halbach type magnetic rotors have been developed for magnetic bearings and motors in the literature [18-20]. Although the basic design of the system is adopted from [7], the detailed design is different. Contrary to [7], the HMBs in this study are placed outside of PMBs (from top to bottom: HMB-PMB-PMB-HMB), which can produce more control torque than the design in [7]. In addition, only one layer of coil is designed for the HMB here. More importantly, the modeling and control issues, and hence the feasibility issues, were not discussed in [7].

The objectives of this study are to establish the magnetic force models for both PMB and HMB in the FESS, and to verify the results experimentally. It is emphasized that since the focus of the paper is on the magnetic force modeling and static levitation of magnetic bearing, only the case without rotation is considered. The most important issue in designing the FESS with Halbach array is the establishment of magnetic force models for both PMB and HMB. Without the magnetic force model, 
several design parameters cannot be determined, such as the geometric sizes of the permanent magnets in the magnetic bearings and the relative position of inner and outer Halbach arrays (which is called offset). More importantly, accurate magnetic force model is the key for obtaining the accurate dynamic model and for designing stable levitation controller. However, establishing accurate magnetic force model is a nontrivial task since it involves many factors, such as magnet geometry, air gap, coil currents, etc.

Although there have been many studies on the magnetic force model of PMB and HMB in the literature [21-25], only few are dealing with PMB and HMB with Halbach array configuration [23-25]. To the best knowledge of the authors, almost no analytic magnetic force model for such system was presented in the literature, especially for the ring-type structure. Most existing models are numerical or in an integral form. Such models are not suitable for the controller design. In this study, we will establish the magnetic force model as analytic algebraic functions of rotor position and coil currents, which are necessary for the controller design.

In this study, to obtain the force model of the overall system, the force model of small magnetic units will be considered first. The complete magnet ring will be approximated by integrating several small magnetic units. Two types of magnetic units are considered: hybrid magnetic unit and passive magnetic unit. The hybrid one consists of electric coil and Halbach array permanent magnet set. It will be used to form HMB. On the other hand, the passive magnetic unit consists of a pair of Halbach array permanent magnet sets with an offset. It will be used to form PMB. The offset of inner and outer Halbach arrays will be chosen so that the PMB can generate the exact vertical magnetic force to support the rotor weight. Hence, the offset can be determined when we have the magnetic force model. The force model of small magnetic units will be established by experimental data. It will be shown that with proper magnet design and coil winding scheme, the magnetic forces of both PMB and HMB can be modeled as linear functions in the relevant variables. The obtained magnetic force models will then be used to obtain the dynamic model of the overall system. It is very difficult, if not impossible, to verify the magnetic force model by direct measurements of the complete HMB and PMB rings due to the inherent instability of the magnetic bearing. In this paper, the force models are verified indirectly. A stable levitation controller is designed based on the obtained models. The good performance of the controller will verify the correctness of the magnetic force models. The results will be verified experimentally.

The paper focuses on the modeling and control of magnetic bearings in the ring-type FESS. The contributions of the paper include:

- A low energy consumption ring-type FESS is proved to be feasible. The ring-type flywheel possesses higher moment of inertia and allows for higher energy storage.

- Magnetic force model of the ring-type HMB with Halbach array configuration is established.

- Magnetic force model of the ring-type PMB with Halbach array configuration is established.

It is emphasized that the magnetic force models presented here are analytical, although they are obtained with the help of experimental data of small magnetic units. Such analytic models allow us to design high performance (advanced and maybe sophisticated) controller. They can also be used for subsequent dynamic analysis, and for the generalization of the prototype system to larger scale.

This paper is organized as follows. After the introduction, the magnetic force models of magnetic bearings and governing dynamic equation of the overall system are described in Section 2. In Section 3, a stabilizing controller for the HMB system is designed using the technique of integral sliding mode control (ISMC). The experimental results are presented in Section 4. Finally, conclusions are drawn in Section 5 . 


\section{System Modeling}

\subsection{Description of Flywheel Energy Storage System}

Figure 1 is the ring-type FESS under study. The inner and outer diameters of the rotor ring are, respectively, $112 \mathrm{~mm}$ and $240 \mathrm{~mm}$, with height of $213 \mathrm{~mm}$. The designed specifications of the prototype FESS are: rated operation speed of 10,000 rpm, rated power of $500 \mathrm{~W}$, stored energy of $98.31 \mathrm{~kJ}$, volumetric energy density of $1.37 \times 104 \mathrm{~kJ} / \mathrm{m}^{3}$ and specific energy of $4.74 \mathrm{~kJ} / \mathrm{kg}$. The system consists of axial PMB, radial HMB, and back-up ball bearings. Recall that this study focuses on the magnetic force modeling and static levitation of magnetic bearing, and hence the motor is not included here. The locations of PMB and HMB with Halbach array are also indicated in Figure 1. There are two sets of radial HMB, one at the top and the other at the bottom of the FESS, for the active control of four DOFs of the rotor. The schematic of the radial HMB is shown in Figure 2. On the rotor side of HMB, there are three layers of permanent magnet rings arranged in a Halbach array, and the corresponding actuator coils are mounted on the stator side of HMB. The rotor can be actively stabilized by the attraction and repulsion forces provided by the $\mathrm{HMB}$, depending on positive or negative currents. Note that no vertical force will be generated with this configuration.

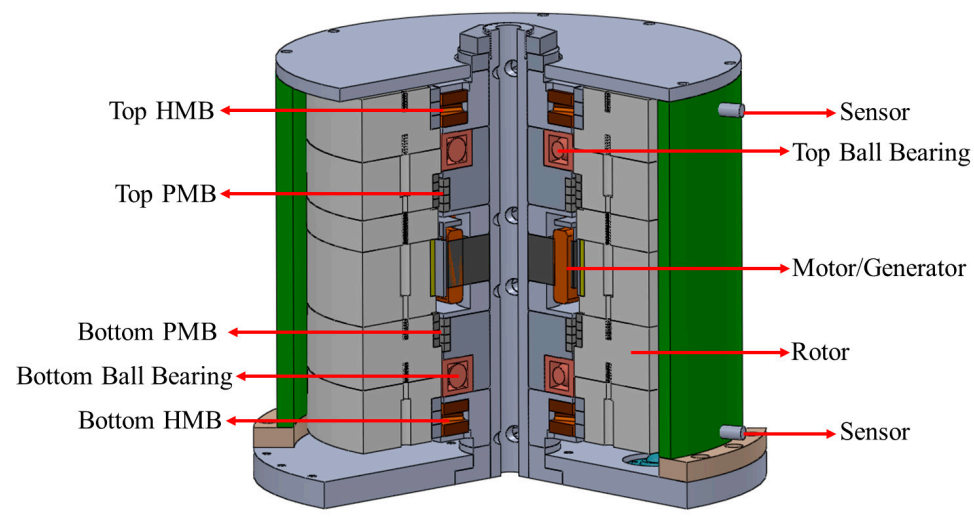

Figure 1. The prototype of flywheel energy storage system (FESS) in this study.

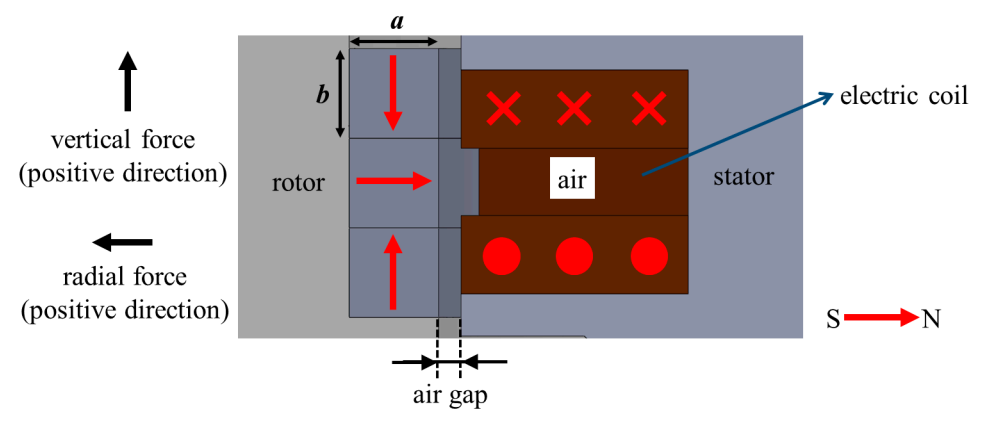

Figure 2. The schematic of the radial hybrid magnetic bearing (HMB).

There are also two sets of PMB, located at upper and lower sides of the motor. The schematic of the axial PMB is shown in Figure 3. On both sides of stator and rotor, there are four layers of permanent magnet rings in Halbach array arrangement. With this configuration, both vertical and radial forces can be generated. The only objective of the PMB is to support the rotor weight in the vertical direction. Thus, a stable (but passive) vertical force is desired, and any radial force is undesirable. For the present design configuration, it is impossible to generate the vertical force without generating the radial force at the same time when the rotor is not at the bearing center. Therefore, the radial force of the PMB has to be compensated by the HMB via active control. 


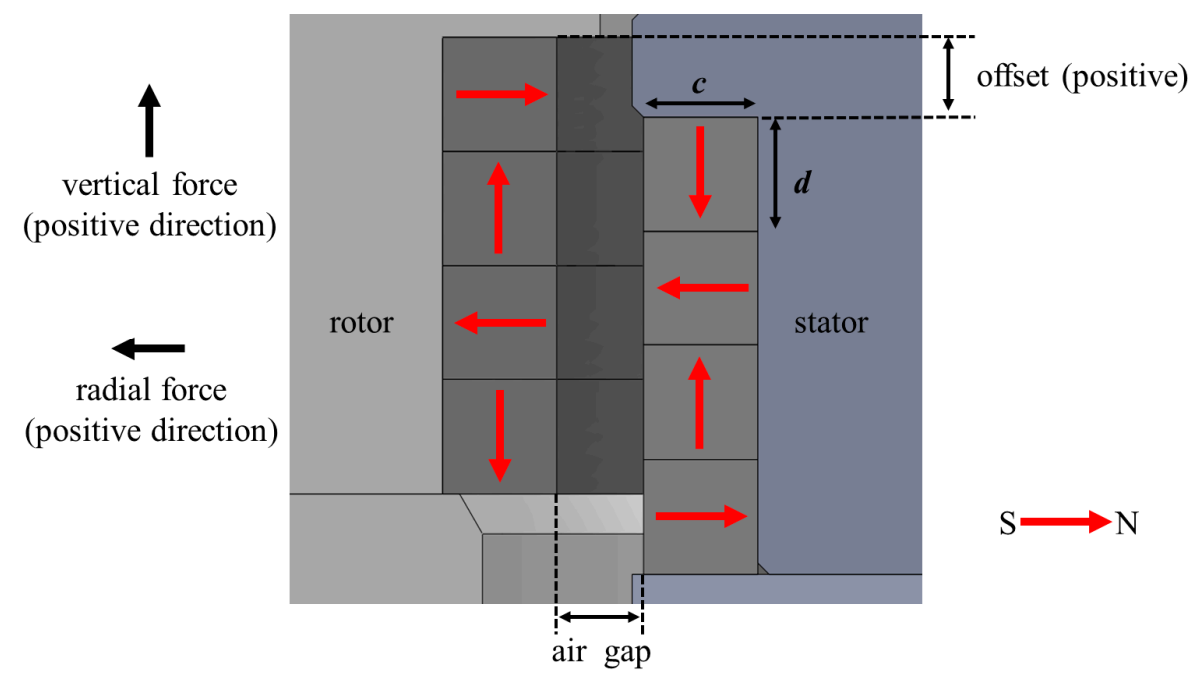

Figure 3. The schematic of the axial passive magnetic bearing (PMB).

\subsection{Hybrid Magnetic Bearing}

In this section, the magnetic force model of HMB will be established. The magnetic force of HMB depends on the coil current and air gap. To identify the relationship, extensive measurements will be conducted. It is difficult to perform the measurements directly on the assembled FESS. The measurements will be performed on a hybrid magnetic unit. Recall that each hybrid magnetic unit consists of a set of electric coils and three layers of Halbach array permanent magnet blocks. Here, the coil is of 350 turns with pole face area of $172.24 \mathrm{~mm}^{2}$, and no iron core in the coil. For the Halbach array, each permanent magnet block is of $8 \mathrm{~mm} \times 8 \mathrm{~mm} \times 30 \mathrm{~mm}(a=8 \mathrm{~mm}$ and $b=8 \mathrm{~mm}$ in Figure 2) with different polarity, and the material is Neodymium magnet (Nd-Fe-B).

The magnetic force is measured by a load cell (LSB302 of Futek Advanced Sensor Technology Inc., Irvine, CA, USA) with measuring range of $-100.0-100.0 \mathrm{~N}$ and resolution of $0.1 \mathrm{~N}$. Figure 4 shows the experimental set-up, where the horizontal position of the test table can be adjusted manually to change the air gap. For each fixed air gap, the generated magnetic forces will be measured with a variety of given coil currents. Because of no iron core, no vertical force can be generated and only the normal force (i.e., the radial force is this study) is measured. The air gap is measured by a laser displacement sensor (LK-G35, Keyence, Osaka, Japan). The measurement results are shown in Figure 5. Three air gaps, $1.5 \mathrm{~mm}, 2.0 \mathrm{~mm}$ and $2.5 \mathrm{~mm}$, with coil currents from -2.0 to $2.0 \mathrm{~A}$ are taken. The results indicate that for each hybrid magnetic unit, the magnetic force is linearly proportional to the coil current. In other words, we have:

$$
f_{h} \approx k_{h}(s) i
$$

where $s$ is the air gap, $i$ is the coil current, and $k_{h}$ is a function of the air gap. The subscript " $h$ " is referred to hybrid magnetic force. Note that positive $f_{h}$ denotes repulsion force and negative $f_{h}$ denotes attraction force. In this study, the function $k_{h}$ is approximated by a 4th degree of polynomial in the air gap. In other words, there are five coefficients for the function, which can be obtained by least-square curve fitting. The root mean squared error of the fitted curve is $0.13 \mathrm{~N}$, and the corresponding $R^{2}$ value is 0.9997 , indicating the excellent goodness of the fit. The results reveal different characteristic of the force model from the conventional electromagnet, where the force is proportional to the square of coil current and inversely proportianal to the square of air gap. 


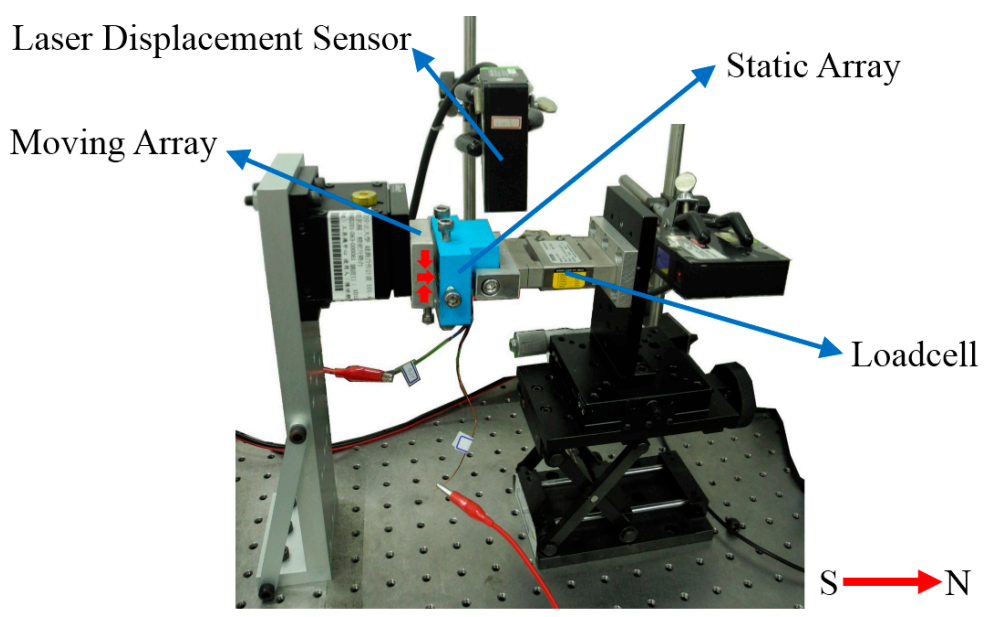

Figure 4. The measurement setup for a hybrid magnetic unit.

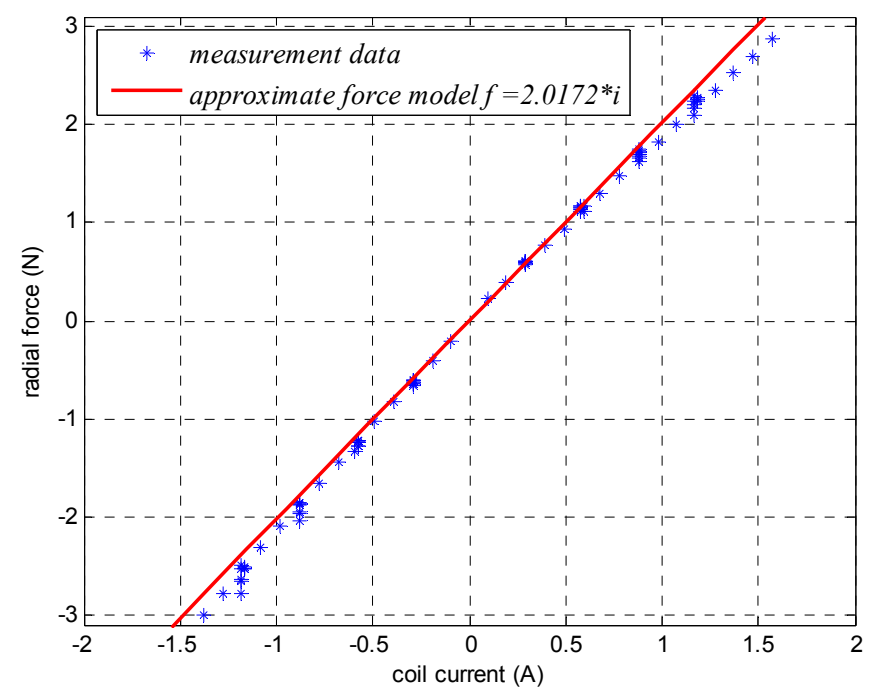

(a)

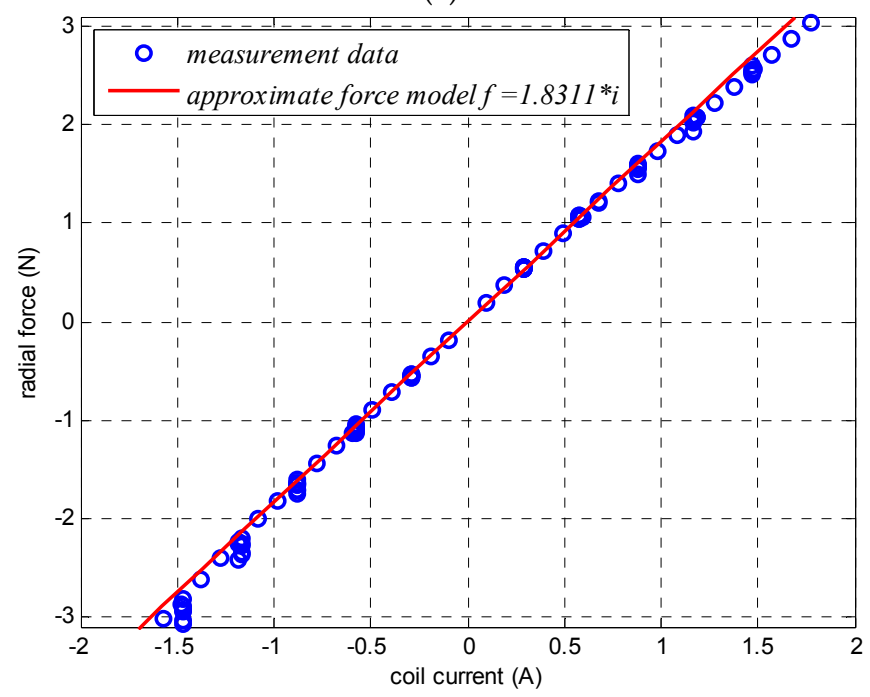

(b)

Figure 5. Cont. 


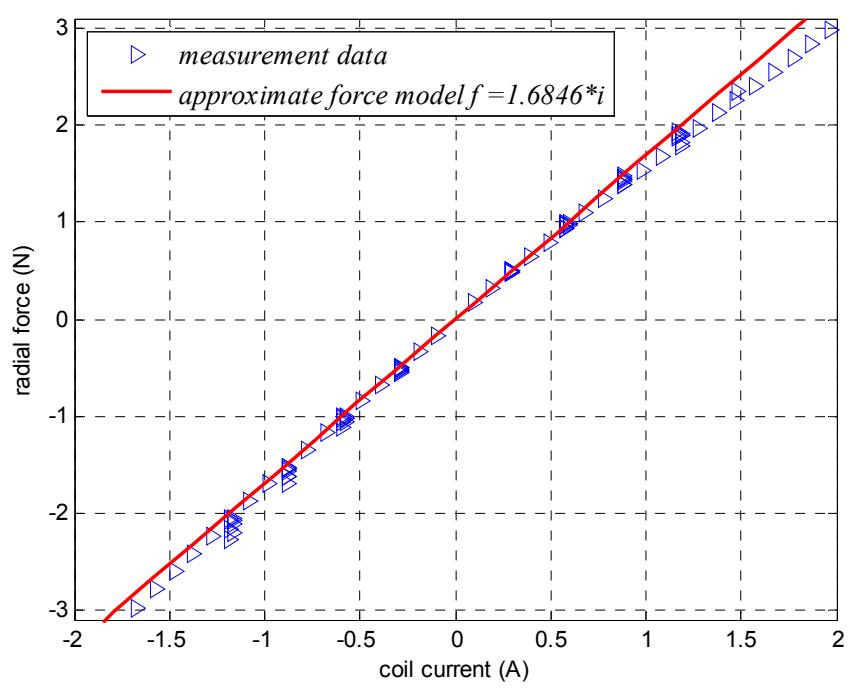

(c)

Figure 5. The magnetic force of a hybrid magnetic unit $(N=350)$ : (a) $s=1.5 \mathrm{~mm}$; (b) $s=2.0 \mathrm{~mm}$; and (c) $s=2.5 \mathrm{~mm}$.

A complete HMB is formed by eight sets of hybrid magnetic units described above, with slight modification. In the experimental prototype, the hybrid magnetic units are arc-shaped in order to form a circular shape. The winding scheme of the HMB is shown in Figure 6. The eight coil sets on the stator (inner part) are grouped in four pairs. Two pairs of coil sets (apart by $180^{\circ}$ ) are wired in a differential way. As a result, there are two independent control currents in each $\mathrm{HMB}$, controlling two DOFs in a horizontal plane. As mentioned above, we have established the magnetic force model (1) for each hybrid magnetic unit through experimental measurements. To obtain the magnetic force model for the overall HMB, the eight arc-shaped hybrid magnetic units in Figure 6 are approximately modeled as eight cuboid hybrid magnetic units, as shown in Figure 7. In other words, there are eight magnetic forces distributed along the radial direction. These force functions are all in the form of Equation (1). Although it is quite nonlinear in the air gap for each magnetic force, the resultant forces of the HMB in both $X$ and $Y$ directions turn out to be independent of the air gap. In other words, the resultant magnetic forces can be modeled as:

$$
\begin{aligned}
& f_{h t x} \approx \sum_{i=1}^{8} f_{h t i} \cos \phi_{i} \approx k_{1} i_{t x} \\
& f_{h t y} \approx \sum_{i=1}^{8} f_{h t i} \sin \phi_{i} \approx k_{2} i_{t y} \\
& f_{h b x} \approx \sum_{i=1}^{8} f_{h b i} \cos \phi_{i} \approx k_{3} i_{b x} \\
& f_{h b y} \approx \sum_{i=1}^{8} f_{h b i} \sin \phi_{i} \approx k_{4} i_{b y}
\end{aligned}
$$

where the subscripts " $t$ " and " $b$ " denote top and bottom, respectively. That is, $f_{h t x}$ represents the resultant magnetic force in the $X$ direction at the top HMB that can be used for active control. Similar definitions apply to other magnetic forces here and in the sequel. In addition, $\phi_{i}$ is angle between the $i$-th hybrid magnetic unit and $X$-axis. In addition, $i_{t x}, i_{t y}, i_{b x}, i_{b y}$ are control currents and $k_{1}, k_{2}, k_{3}$, $k_{4}$ are constants. The common differential winding scheme in AMB will provide a magnetic force as function of displacement and current in a single linearized equation around the operating point. Note 
here that the magnetic forces generated by the HMB depend only on the control currents, not on the rotor displacements because of the winding scheme and no iron core used in the HMB, so that, no matter where the rotor position is, there is no interaction between rotor and stator as long as the coil current is zero.

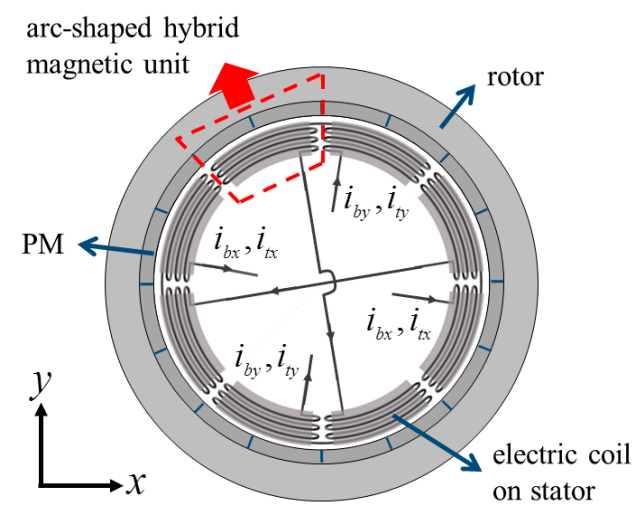

Figure 6. The winding scheme of HMB.

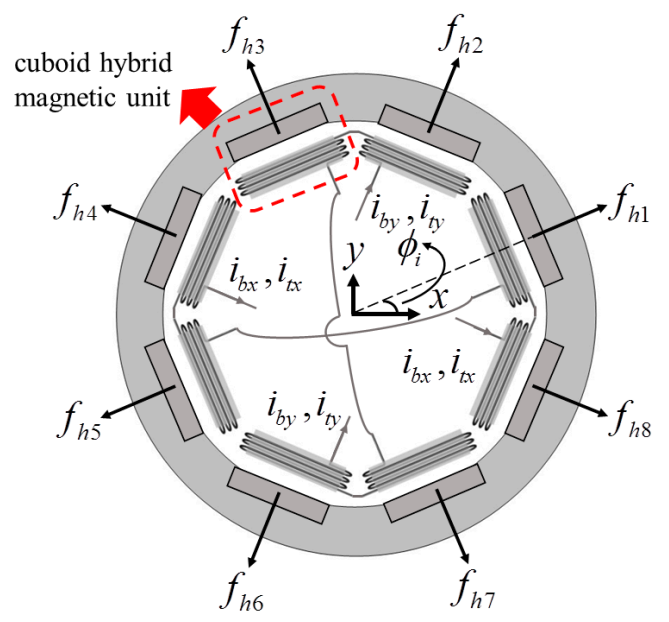

Figure 7. An octagon structure with eight hybrid magnetic units.

\subsection{Passive Magnetic Bearing}

Next, the magnetic force model of PMB will be established. For the PMB, the magnetic forces are functions of the air gap and offset. To identify the force model, extensive measurements were conducted. Similar to the case of HMB, the measurements will be performed on a single passive magnetic unit. For the Halbach array, each permanent magnet block is of $5 \mathrm{~mm} \times 5 \mathrm{~mm} \times 30 \mathrm{~mm}$ ( $c=5 \mathrm{~mm}$ and $d=5 \mathrm{~mm}$ in Figure 3) with different polarity, and the material is bonded NdFeB magnet, which is made by mixing rare earth powder and resin, and is formed by compression. Figure 8 is the experimental set-up, where both the horizontal and vertical positions of the test table can be adjusted manually to change the air gap and offset, respectively. For each fixed air gap, both the normal and vertical magnetic forces will be measured with a variety of offsets. Both the air gap and offset will be measured by laser displacement sensors. Some of the measurement results for a single passive magnetic unit are presented in Figure 9, where the solid and blue lines represent the vertical forces, and the dashed and red lines are the radial forces.

The radial and vertical magnetic forces generated by a single passive magnetic unit are assumed to be 5th degree of polynomial functions in the air gap and offset as:

$$
f_{p r} \approx k_{p r}(z, s), f_{p v} \approx k_{p v}(z, s)
$$


where $z$ is offset, and the subscript " $p$ " denotes passive and subscript " $r$ " and " $v$ " denotes radial and vertical, respectively. In other words, there are 21 coefficients for each function. From the experimental data, one can determine the coefficients by the least square curve fitting. The root mean squared errors of the fitted curves are $0.64 \mathrm{~N}$ and $0.87 \mathrm{~N}$ for vertical and radial forces, respectively. The corresponding $R^{2}$ values for the fitted vertical and radial force functions are 0.9915 and 0.9819 , respectively. Again, the results indicate the excellent goodness of the fit.

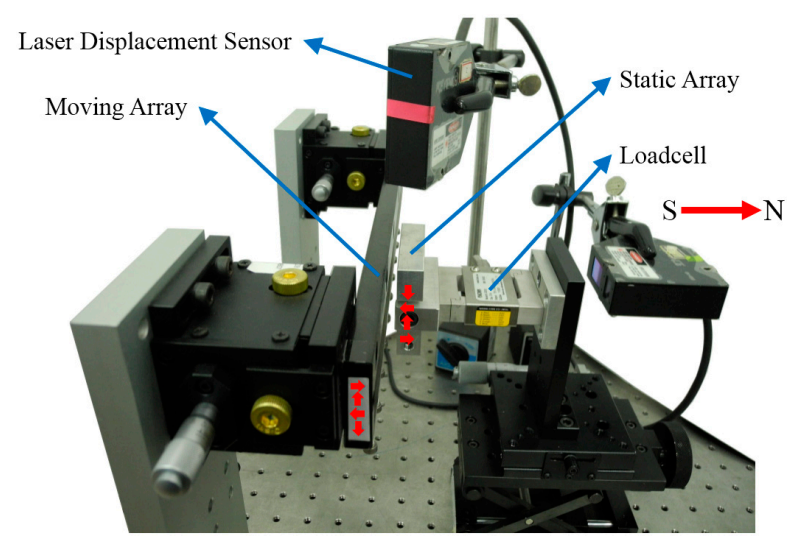

Figure 8. The setup of measurement for a passive magnetic unit.

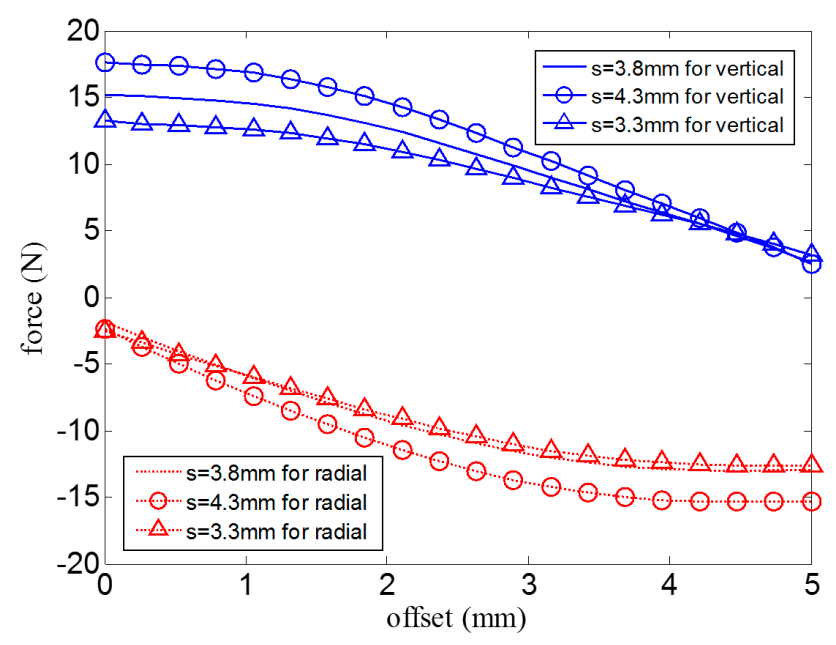

Figure 9. The magnetic force of a passive magnetic unit.

A complete PMB is formed by 16 sets of passive magnetic units described above, as shown in Figure 10. These functions are all in the form of Equation (6). The resulting magnetic forces of two PMB on vertical direction for three different air gaps are shown in Figure 11. The main function of PMB is to support the rotor weight, which will be achieved by the integrated vertical force. Note that the rotor weight (about $204 \mathrm{~N}$ ) is also indicated in Figure 11. From Figure 11, the operation point must be located at a position offset so that the vertical force can balance the rotor weight. In the experimental prototype, the nominal air gap is designed to be $s=3.8 \mathrm{~mm}$; hence, the offset of $z=3.4 \mathrm{~mm}$ should be taken. Moreover, at this operation point, the axial motion is passively stabilized with stiffness of $84.22 \mathrm{KN} / \mathrm{m}$. 


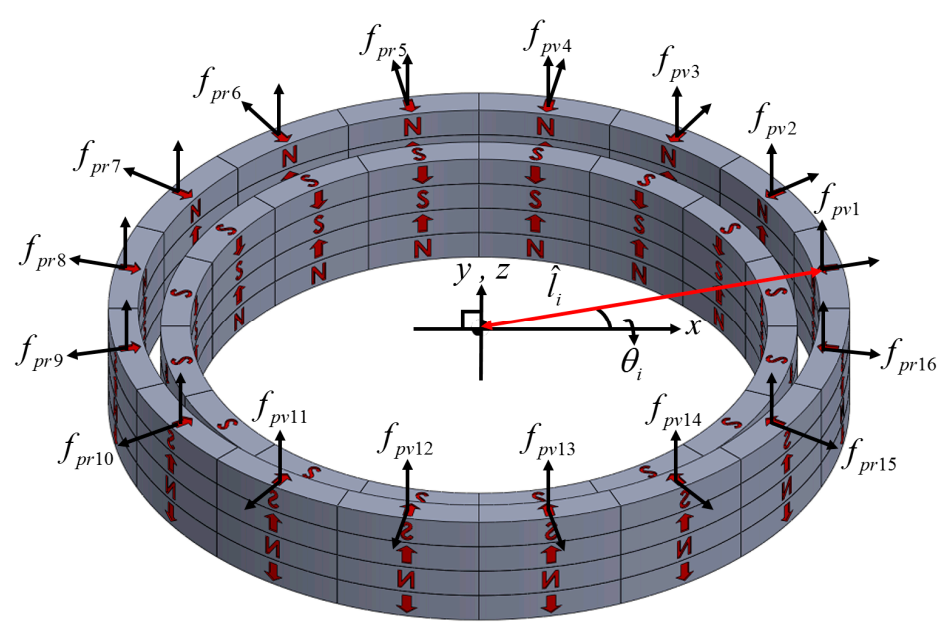

Figure 10. The moments caused by the vertical force $f_{p v}$ of 16 sets of passive magnetic units about $X$-axis and $Y$-axis.

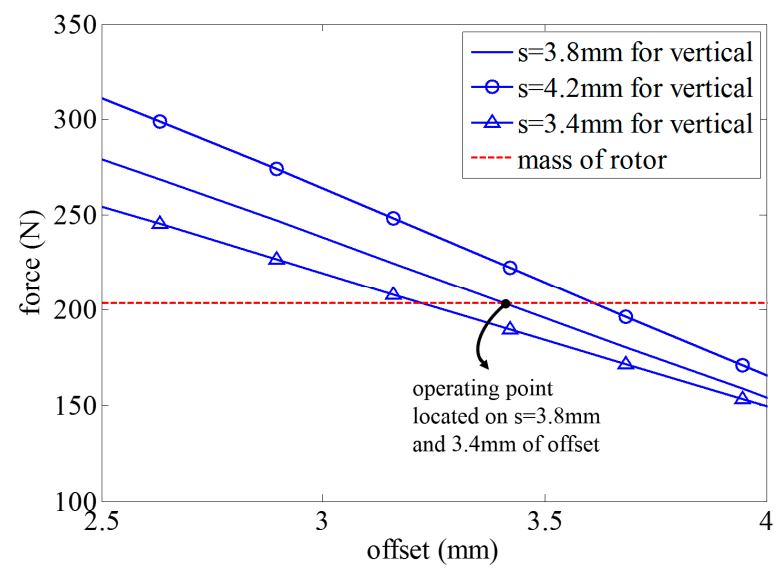

Figure 11. The resulting magnetic forces of PMB on vertical direction.

In addition to supporting the rotor weight, the vertical force will also induce moments to the rotor when the rotor is not at the center position. This will cause undesirable tilting rotation motion, and needs to be compensated by the HMB. To this aim, it is desired to express the force model as a function of rotor displacements at the location of HMB. Let $x_{t}, x_{b}, y_{t}$ and $y_{b}$ be the rotor displacements at the location of HMB. Let the moments caused by the vertical force of top HMB and bottom HMB be denoted by $M_{v x}$ and $M_{v y}$. They are contributed from the 16 sets of passive magnetic units shown in Figure 10. Although the vertical force of each passive magnetic unit is quite nonlinear, the integrated moments $M_{v x}$ and $M_{v y}$ can be modeled as linear functions of the rotor displacements at the location of $\mathrm{HMB}$, and it can expressed as:

$$
\begin{aligned}
& M_{v x} \approx \sum_{i=1}^{16}\left[f_{p v t i}\left(z, s_{t i}\right) \hat{l}_{t i}\left(s_{t i}\right)+f_{p v b i}\left(z, s_{b i}\right) \hat{l}_{b i}\left(s_{b i}\right)\right] \sin \theta_{i} \approx-k_{m y t} y_{t}-k_{m y b} y_{b}, \\
& M_{v y} \approx-\sum_{i=1}^{16}\left[f_{p v t i}\left(z, s_{t i}\right) \hat{l}_{t i}\left(s_{t i}\right)+f_{p v b i}\left(z, s_{b i}\right) \hat{l}_{b i}\left(s_{b i}\right)\right] \cos \theta_{i} \approx k_{m x t} x_{t}+k_{m x b} x_{b},
\end{aligned}
$$

where $f_{\text {pvti }}\left(z, s_{t i}\right)$ denotes the passive vertical magnetic force at the top location contributed from the $i$ th unit, $\hat{l}_{t i}\left(s_{t i}\right)$ is distance between the force and the rotor's mass center on plane of top PMB, and $s_{t i}$ is the air gap at the top location of the $i$ th unit, which is a function of $x_{t}, x_{b}, y_{t}$, and $y_{b}$. Similar definitions 
apply to other magnetic forces here and in the sequel. In addition, $\theta_{i}$ represents the angle between the $i$ th passive magnetic unit and $X$-axis on $X-Y$ plane as shown in Figure 10.

Note that there are 16 magnetic forces distributed along the circumference as shown in Figure 12. The radial forces generated by the PMB are also undesirable, and are to be compensated by the HMB. For a given offset (at the operation point), the radial force is a function of the air gap, which is in turn a function of the rotor displacement $x_{t}, x_{b}, y_{t}$ and $y_{b}$. From Equation (6), one can see that although radial force is quite nonlinear in the air gap for each magnetic unit, the integrated forces of the PMB in both $X$ and $Y$ directions can be modeled as linear functions of the rotor displacements at the location of HMB. In other words, the resultant radial magnetic forces can be modeled as:

$$
\begin{gathered}
f_{p t x} \approx \sum_{i=1}^{16} f_{p r t i} \cos \left(\theta_{i}\right) \approx k_{5} x_{t}+k_{6} x_{b}, \\
f_{p t y} \approx \sum_{i=1}^{16} f_{p r t i} \sin \left(\theta_{i}\right) \approx k_{7} y_{t}+k_{8} y_{b}, \\
f_{p b x} \approx \sum_{i=1}^{16} f_{p r b i} \cos \left(\theta_{i}\right) \approx k_{9} x_{t}+k_{10} x_{b}, \\
f_{p b y} \approx \sum_{i=1}^{16} f_{p r b i} \sin \left(\theta_{i}\right) \approx k_{11} y_{t}+k_{12} y_{b},
\end{gathered}
$$

where $k_{5}, k_{6}, k_{7}, k_{8}, k_{9}, k_{10}, k_{11}$ and $k_{12}$ are constants.

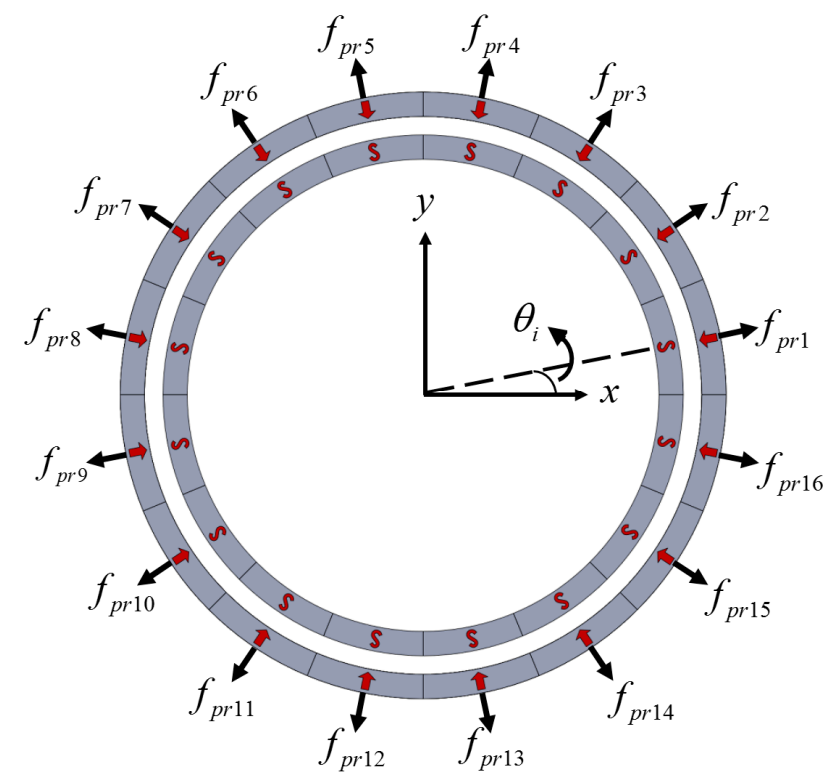

Figure 12. A top-view on PMB and the radial force for 16 sets of passive magnetic units.

\subsection{Governing Equation}

With the magnetic force model, we can now establish the equations of motion for the overall FESS. As mentioned previously, this study focuses on the magnetic force modeling and stable levitation of magnetic bearing, and only the case without rotation is considered. Since the motion in the Z-direction is passively stabilized with high stiffness, it is assumed that the rotor displacements in the Z-direction are very small and only the dynamics of four DOFs are considered. By Newton's law, one can easily obtain the equations of motion as:

$$
\sum F_{x}=m \frac{l_{t} \ddot{x}_{b}+l_{b} \ddot{x}_{t}}{l_{t}+l_{b}}
$$




$$
\begin{gathered}
\sum F_{y}=m \frac{l_{t} \ddot{y}_{b}+l_{b} \ddot{y}_{t}}{l_{t}+l_{b}}, \\
\sum M_{x}=J_{t} \frac{\ddot{y}_{b}-\ddot{y}_{t}}{l_{b}+l_{t}}, \\
\sum M_{y}=J_{t} \frac{\ddot{x}_{t}-\ddot{x}_{b}}{l_{b}+l_{t}},
\end{gathered}
$$

and,

$$
\begin{gathered}
\sum F_{x}=k_{1} i_{t x}+k_{3} i_{b x}+k_{13} x_{t}+k_{14} x_{b}, \\
\sum F_{y}=k_{2} i_{t y}+k_{4} i_{b y}+k_{15} y_{t}+k_{16} y_{b}, \\
\sum M_{x}=k_{4} i_{b y} l_{b}-k_{2} i_{t y} l_{t}+k_{17} y_{t}+k_{18} y_{b}, \\
\sum M_{y}=k_{1} i_{t x} l_{t}-k_{3} i_{b x} l_{b}+k_{19} x_{t}+k_{20} x_{b}, \\
k_{13}=k_{5}+k_{9}, k_{14}=k_{6}+k_{10}, \\
k_{15}=k_{7}+k_{11}, k_{16}=k_{8}+k_{12}, \\
k_{17}=k_{11} l_{b p}-k_{7} l_{t p}-k_{m y t}, k_{18}=k_{12} l_{b p}-k_{8} l_{t p}-k_{m y b}, \\
k_{19}=k_{5} l_{t p}-k_{9} l_{b p}+k_{m x t}, k_{20}=k_{6} l_{t p}-k_{10} l_{b p}+k_{m x b},
\end{gathered}
$$

where the superscript "." denotes differentiation with respect to time. Note that $l_{b}$ and $l_{t}$ are nominal distances from mass center to the bottom and top HMB, respectively; $l_{b p}$ and $l_{t p}$ are nominal distances from mass center to the bottom and top PMB, respectively; and $m$ is the rotor mass. In addition, the cross-section radial mass moment of inertia is denoted as $J_{t}$. From Equations (13)-(16), it is obvious that $\mathrm{HMB}$ requires no bias current theoretically if the rotor is stabilized at the center position (i.e., $\left.x_{t}=0, x_{b}=0, y_{t}=0, y_{b}=0\right)$.

\section{Controller Design}

\subsection{State Space Model}

To verify the magnetic force models established in the previous section, a stabilizing controller will be designed in this section based on the obtained force models. Without rotation as assumed in this work, the FESS is of five DOFs. The axial motion will be passively stabilized by the PMB and its dynamics are decoupled from the radial motions. Hence, only the four DOFs radial motion are considered here. From equations of motion given by Equations (13)-(24), the state space model of the overall system can be expressed as:

$$
\dot{\mathbf{x}}=\mathbf{A} \mathbf{x}+\mathbf{B} \mathbf{u}=\left[\begin{array}{cc}
0 & \mathbf{I} \\
\mathbf{A}_{\mathbf{1}} & 0
\end{array}\right] \mathbf{x}+\left[\begin{array}{c}
0 \\
\mathbf{B}_{\mathbf{1}}
\end{array}\right] \mathbf{u},
$$

where $\mathbf{x}=\left[\begin{array}{llllllll}x_{b} & y_{b} & x_{t} & y_{t} & \dot{x}_{b} & \dot{y}_{b} & \dot{x}_{t} & \dot{y}_{t}\end{array}\right]^{T}$ is the state vector, $\mathbf{u}=\left[\begin{array}{llll}i_{b x} & i_{b y} & i_{t x} & i_{t y}\end{array}\right]^{T}$ is the control current, and,

$$
\mathbf{A}_{\mathbf{1}}=\left[\begin{array}{cccc}
-\frac{k_{20} l_{b}}{J_{t}}+\frac{k_{14}}{m} & 0 & -\frac{k_{19} l_{b}}{J_{t}}+\frac{k_{13}}{m} & 0 \\
0 & \frac{k_{18} l_{b}}{J_{t}}+\frac{k_{16}}{m} & 0 & \frac{k_{17} l_{b}}{J_{t}}+\frac{k_{15}}{m} \\
\frac{k_{20} l_{t}}{J_{t}}+\frac{k_{14}}{m} & 0 & \frac{k_{19} l_{t}}{J_{t}}+\frac{k_{13}}{m} & 0 \\
0 & -\frac{k_{18} l_{t}}{J_{t}}+\frac{k_{16}}{m} & 0 & -\frac{k_{17} l_{t}}{J_{t}}+\frac{k_{15}}{m}
\end{array}\right],
$$




$$
\mathbf{B}_{1}=\left[\begin{array}{cccc}
k_{3}\left(\frac{1}{m}+\frac{l_{b}^{2}}{J_{t}}\right) & 0 & k_{1}\left(\frac{1}{m}-\frac{l_{b} l_{t}}{J_{t}}\right) & 0 \\
0 & k_{4}\left(\frac{1}{m}+\frac{l_{b}^{2}}{J_{t}}\right) & 0 & k_{2}\left(\frac{1}{m}-\frac{l_{b} l_{t}}{J_{t}}\right) \\
k_{3}\left(\frac{1}{m}-\frac{l_{b} l_{t}}{J_{t}}\right) & 0 & k_{1}\left(\frac{1}{m}+\frac{l_{t}^{2}}{J_{t}}\right) & 0 \\
0 & k_{4}\left(\frac{1}{m}-\frac{l_{b} l_{t}}{J_{t}}\right) & 0 & k_{2}\left(\frac{1}{m}+\frac{l_{t}^{2}}{J_{t}}\right)
\end{array}\right]
$$

\subsection{Integral Sliding Mode Control}

The mathematical model is based on the approximated force models. There may exist uncertainty in the dynamic model. Thus, it is assumed that the real system model is a nominal model plus a bounded uncertain part. Hence, a robust controller is preferred for practical implementation. In this paper, an integral sliding mode controller will be designed to allow for large uncertainties and to achieve good steady-state accuracy. To facilitate the controller design, the system's dynamic model is rewritten in the regular form as:

$$
\begin{gathered}
\dot{\boldsymbol{\eta}}=\xi, \\
\dot{\xi}=\mathbf{G}_{\mathrm{A}} \mathbf{x}+\mathbf{B}_{\mathbf{1}} \mathbf{u}+\Delta(\mathbf{x}, \mathbf{u}),
\end{gathered}
$$

and $\eta=\left[\begin{array}{llll}x_{1} & x_{2} & x_{3} & x_{4}\end{array}\right]^{T}, \boldsymbol{\xi}=\left[\begin{array}{llll}x_{5} & x_{6} & x_{7} & x_{8}\end{array}\right]^{T}$. Moreover, $\Delta(\mathbf{x}, \mathbf{u})$ represents the uncertainty, and,

$$
\mathbf{G}_{\mathbf{A}}=\left[\begin{array}{ll}
\mathbf{A}_{1} & 0
\end{array}\right] \text {. }
$$

The ISMC takes an integral sliding variable as:

$$
\boldsymbol{\sigma}=\boldsymbol{\xi}+b_{1} \boldsymbol{\eta}+b_{2} \mathbf{z}
$$

and $\mathbf{z}=\int \eta \mathrm{d} t$. Here, $b_{1}$ and $b_{2}$ are positive constants. If the system state is on the sliding manifold $\boldsymbol{\sigma}=0$ and can be maintained on the manifold $(\dot{\boldsymbol{\sigma}}=0)$, the system dynamics will be like:

$$
\ddot{\eta}+b_{1} \dot{\boldsymbol{\eta}}+b_{2} \boldsymbol{\eta}=0,
$$

implying that the system state $\eta$ and $\xi$ will approach zero asymptotically.

The ISMC consists of an equivalent control $\mathbf{u}_{\mathbf{e q}}$ and a switching control $\mathbf{u}_{\mathbf{s}}$. The equivalent control is to maintain the system state on the sliding manifold once it is there, assuming no uncertainty. The switching control is to bring the system state to the sliding manifold in finite time, with the presence of uncertainty. Following the standard design procedure [26], one can get:

$$
\mathbf{u}=\mathbf{u}_{\mathrm{eq}}+\mathbf{u}_{\mathbf{s}}
$$

where:

$$
\begin{aligned}
& \mathbf{u}_{\mathrm{eq}}=\mathbf{B}_{\mathbf{1}}^{-1}\left(-\mathbf{G}_{\mathbf{A}} \mathbf{x}-b_{1} \boldsymbol{\xi}-b_{2} \boldsymbol{\eta}\right), \\
& \mathbf{u}_{\mathbf{s}}=-k_{c} \mathbf{B}_{1}^{-1} \tanh (\sigma, \varepsilon), \\
& \tanh (\boldsymbol{\sigma}, \varepsilon)=\left[\begin{array}{llll}
\tanh \left(\frac{\boldsymbol{\sigma}_{1}}{\varepsilon}\right) & \tanh \left(\frac{\boldsymbol{\sigma}_{2}}{\varepsilon}\right) & \tanh \left(\frac{\boldsymbol{\sigma}_{3}}{\varepsilon}\right) & \tanh \left(\frac{\boldsymbol{\sigma}_{4}}{\varepsilon}\right)
\end{array}\right]^{T}
\end{aligned}
$$

From Equation (27), it is easy to see that $\mathbf{B}_{\mathbf{1}}$ is invertible. Here, the smooth type switching function $\tanh (\sigma, \varepsilon)$ is used, $\sigma_{i}$ are the components of the sliding variable $\sigma$, and $k_{c}$ and $\varepsilon$ are positive constants.

\section{Experimental Results}

A prototype of FESS has been manufactured to verify the magnetic force models established in Section 2 and the levatation controller designed in Section 3. The fabrication and magnetization of the 
ring-type Halbach array magnet is very difficult since it is thin $(5 \mathrm{~mm})$. As described in Section 3, the $\mathrm{PMB}$ and the rotor of HMB are composed of segmented arc-shaped permanent magnets.

Figure 13 is the stator and Figure 14 is the rotor of HMB and PMB. The assembled FESS protytpe is shown in Figure 15. Most of the system is made of aluminum alloy. The rotor is coated with copper for better look. After all of the parts are assembled together, it is easy to find that the lowest part does not touch the back-up bearing on the bottom, showing the successful levitation of the PMB. The system parameters are listed in Table 1, and the parameters of the magnetic bearings are listed in Table 2. With this FESS prototype, the parameters in the magnetic force models are collected in Table 3.

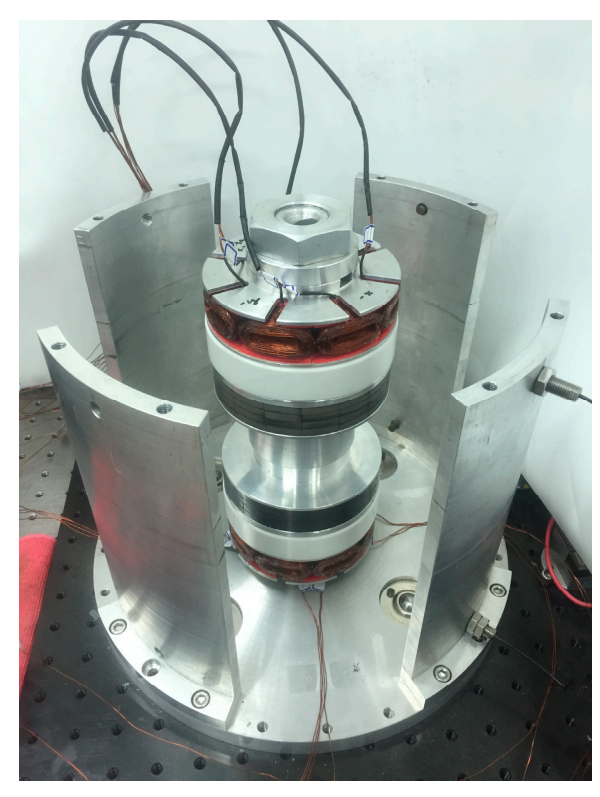

Figure 13. The stator of HMB and PMB.

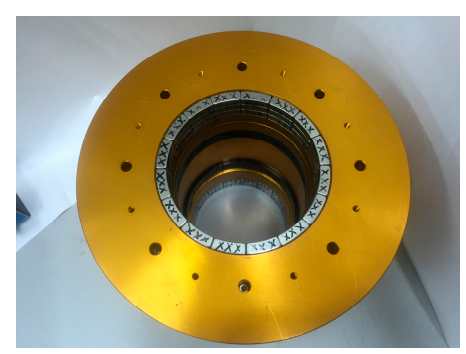

Figure 14. The rotor of HMB and PMB.

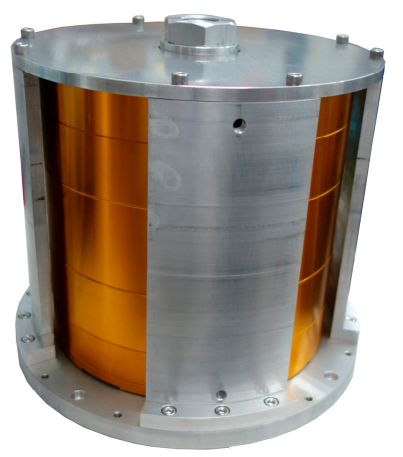

Figure 15. The assembled FESS prototype. 
Table 1. Parameters of proposed FESS prototype.

\begin{tabular}{ccc}
\hline Symbol & Quantity & Value \\
\hline$m$ & mass of rotor & $20.76 \mathrm{~kg}$ \\
$J_{t}$ & cross-section radial mass moments of inertia & $0.1720 \mathrm{~kg} \cdot \mathrm{m}^{2}$ \\
$J_{p}$ & polar mass moments of inertia & $0.1793 \mathrm{~kg} \cdot \mathrm{m}^{2}$ \\
$l_{b}$ & nominal distances from mass center to the bottom HMB & $92.97 \mathrm{~mm}$ \\
$l_{t}$ & nominal distances from mass center to the top HMB & $93.03 \mathrm{~mm}$ \\
$l_{b p}$ & nominal distances from mass center to the bottom and top PMB & $42.12 \mathrm{~mm}$ \\
$l_{t p}$ & nominal distances from mass center to the bottom and top PMB & $41.88 \mathrm{~mm}$ \\
\hline
\end{tabular}

Table 2. Parameters of HMB and PMB.

\begin{tabular}{ccc}
\hline Symbol & Quantity & Value \\
\hline$l_{0 a}$ & nominal air gap of HMB & $2.0 \mathrm{~mm}$ \\
$l_{0 p}$ & nominal air gap of PMB & $3.8 \mathrm{~mm}$ \\
$z_{0}$ & nominal offset of PMB & $3.4 \mathrm{~mm}$ \\
$N$ & number of coil turns & 350 \\
$a$ & width of permanent magnet for HMB & $8.00 \mathrm{~mm}$ \\
$b$ & height of permanent magnet for HMB & $8.00 \mathrm{~mm}$ \\
$c$ & width of permanent magnet for PMB & $5.00 \mathrm{~mm}$ \\
$d$ & height of permanent magnet for PMB & $5.00 \mathrm{~mm}$ \\
\hline
\end{tabular}

Table 3. Parameters of approximate magnetic force.

\begin{tabular}{ccc}
\hline Symbol & Quantity & Value \\
\hline$k_{1}, k_{2}, k_{3}, k_{4}$ & coefficients of AMB & $6.8467 \mathrm{~N} / \mathrm{A}$ \\
$k_{5}, k_{7}$ & coefficients of PMB & $14,787 \mathrm{~N} / \mathrm{m}$ \\
$k_{6}, k_{8}$ & coefficients of PMB & $5494 \mathrm{~N} / \mathrm{m}$ \\
$k_{9}, k_{11}$ & coefficients of PMB & $5460 \mathrm{~N} / \mathrm{m}$ \\
$k_{10}, k_{12}$ & coefficients of PMB & $14,819 \mathrm{~N} / \mathrm{m}$ \\
$k_{m x b}, k_{m y b}$ & coefficients of moments by PMB & $601.18 \mathrm{~N}$ \\
$k_{m x t}, k_{m y t}$ & coefficients of moments by PMB & $599.25 \mathrm{~N}$ \\
\hline
\end{tabular}

The PID controller cannot work due to the uncertainties of force model, which has been verified in numerical simulations. The robustness of ISMC controller is essential in this experiment. Table 4 shows the parameters of the ISMC controller. They are obtained on a trial and error basis using numerical simulations carried out by MATLAB/Simulink (https://www.mathworks.com/products/simulink. html). These controller parameters will also be adopted for the closed-loop experiments without motor. For real-time implementation, the control algorithm is downloaded into dSpace's control card (DS1103, Paderborn, Germany). The pulse-width modulation type power amplifier is utilized to produce appropriate control currents to the coil sets of HMB. Please refer to Figure 16 for experimental set-up. The rotor displacements are measured with eddy current sensors. The position sensors are placed on the horizontal planes passing through the top and bottom HMBs. The measurements of position sensors are used to calculate the rotor position-the displacement of the geometric center of the cylindrical, ring-type rotor. The control objective is to drive the rotor position to coincide with the geometric center of the stator. 
Table 4. Parameters of integral sliding mode controller.

\begin{tabular}{ccc}
\hline Symbol & Quantity & Value \\
\hline$b_{1}$ & parameter of equivalent control & 30.00 \\
$b_{2}$ & parameter of equivalent control & 55.00 \\
$k_{c}$ & parameter of switching control & 56.66 \\
$\varepsilon$ & parameter of switching control & 0.50 \\
\hline
\end{tabular}

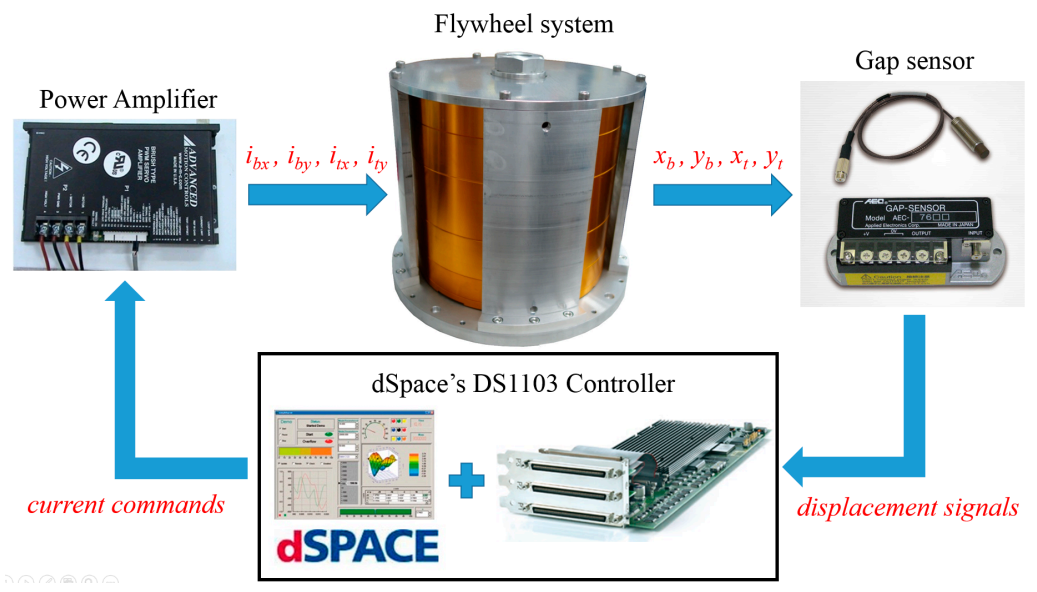

Figure 16. Block diagram of the experiment setup.

Figures 17-19 show the experimental results, i.e., the rotor trajectory, displacements $x_{t}, x_{b}, y_{t}$, and $y_{b}$ and control currents $i_{t x}, i_{t y}, i_{b x}$, and $i_{b y}$. The dashed red curve in Figure 17 represents the allowable range of the rotor displacements formed by the back-up bearing. The experimental results indicate that the rotor can be stabilized to the bearing center with settling time around $0.72-1.06 \mathrm{~s}$ and $20.8 \%-38.9 \%$ overshoot for two HMB, respectively. The steady state errors are all less than $1 \mu \mathrm{m}$, approaching the resolution of the position sensors. The results show the good performance of the levitation controller, and clearly verify the accuracy of the magnetic force models.

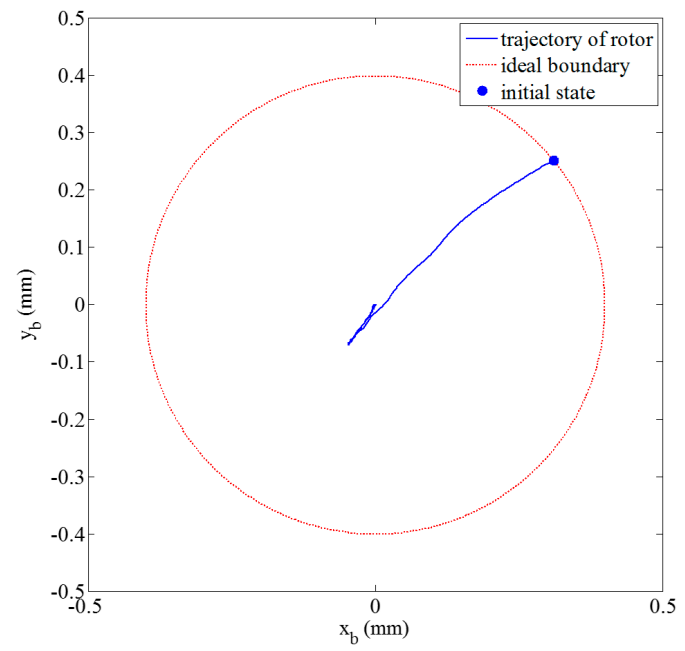

(a)

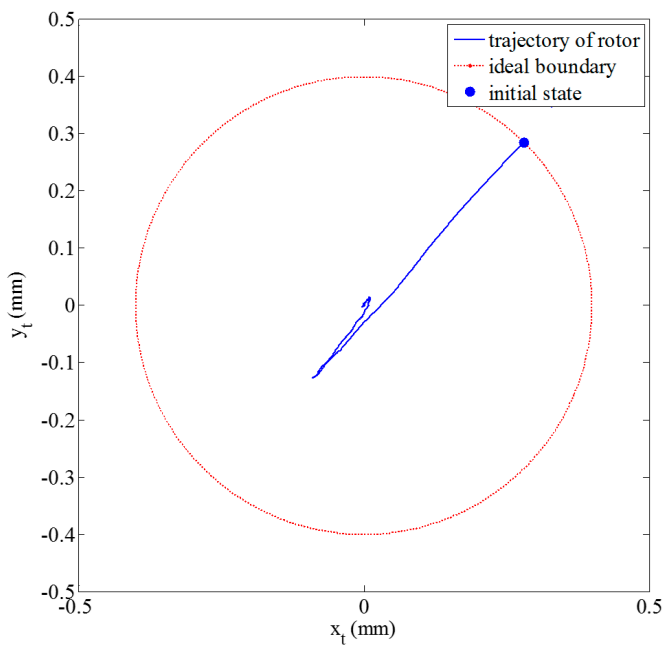

(b)

Figure 17. Experimental results: (a) rotor trajectory at the bottom HMB; and (b) rotor trajectory at the top HMB. 


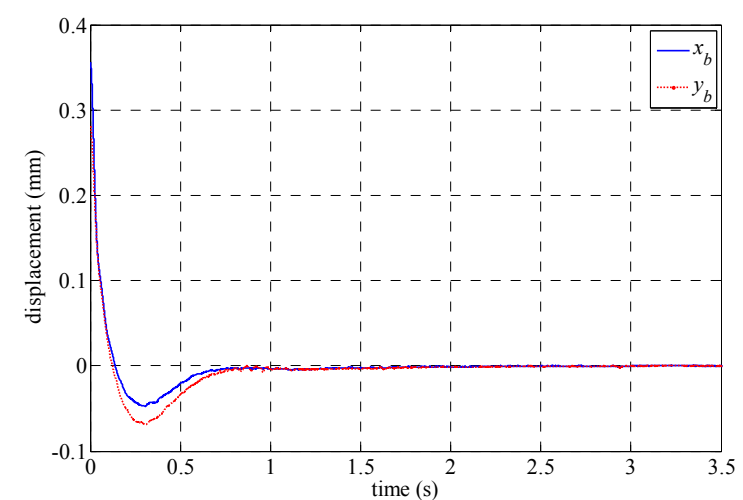

(a)

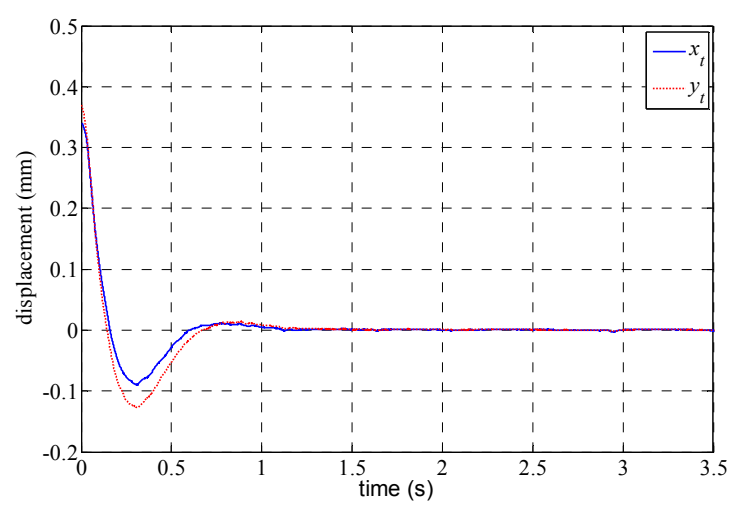

(b)

Figure 18. Experimental results: (a) displacement $y_{b}$ and $y_{b}$; and (b) displacement $x_{t}$ and $y_{t}$.

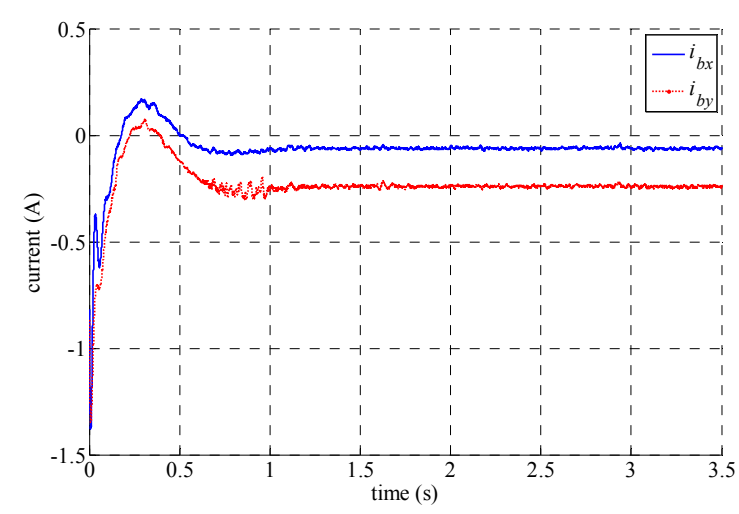

(a)

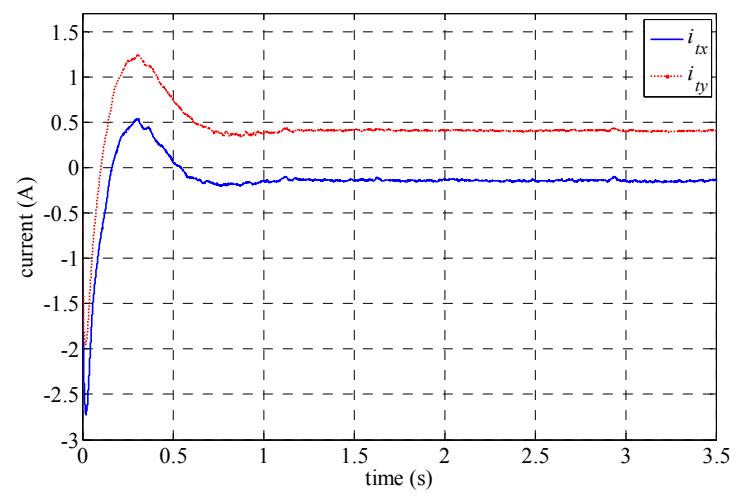

(b)

Figure 19. Experimental results: (a) control currents $i_{b x}$ and $i_{b y}$; and (b) control currents $i_{t x}$ and $i_{t y}$.

Finally, Figure 19 shows the control currents, which are all less than $0.5 \mathrm{~A}$ at the steady state. Theoretically, the steady state control currents should be zero since the rotor is at the bearing center. The nonzero currents here could be due to the non-uniform air gaps between rotor and stator. Such non-uniform air gap can be a result of the manufacturing and assembly errors. The nonzero steady state currents account for only $2.2 \mathrm{~W}$ of energy consumption. Compared to the rated power $500 \mathrm{~W}$ of the FESS, the energy loss is low and acceptable. In practical applications, it is expected that more accuracy of manufacturing and assembly can be achieved. Thus, the steady state currents can be reduced and hence the energy consumption loss can be further reduced. Compare to the conventional $\mathrm{AMB}$, the experimental results reveal that the low energy consumption HMB is promising in the field of FESS.

\section{Conclusions}

In this study, the magnetic force models of HMB and PMB in a FESS have been established. Both $\mathrm{HMB}$ and PMB contain permanent magnet rings in Halbach array arrangement. It is found that the ring-type configuration and the differential winding scheme for coil sets can effectively simplify the magnetic force models. In particular, for $\mathrm{HMB}$, no vertical force will be generated, and the radial magnetic force is linearly proportional to the coil current and is independent of the air gap. For PMB, both vertical and radial forces will be generated. An operation point of offset and air gap can be determined based on the vertical force model. The radial magnetic force and the moments by the vertical magnetic force can be modeled as linear functions of the rotor displacements. The parameters of the magnetic force models are identified by extensive measurements. To verify the results, a stabilizing 
levitation controller is designed based on the obtained magnetic force models. For robustness, an ISMC controller is designed. The experimental results show that the rotor can be stabilized to the bearing center, confirming the accuracy of the magnetic force model. The robustness of suppressing the external disturbances and model uncertainties, such as assembly errors, manufacturing errors, mass unbalance and sensor noise, at a constant rotor speed will be the further work.

Acknowledgments: This work was supported by the Ministry of Science and Technology of Taiwan, under the Grant MOST 103-2221-E-194-008.

Author Contributions: Chow-Shing Toh and Shyh-Leh Chen conceived and designed the experiments; Chow-Shing Toh performed the experiments; Chow-Shing Toh analyzed the data; Chow-Shing Toh and Shyh-Leh Chen wrote the paper.

Conflicts of Interest: The authors declare no conflict of interest.

\section{References}

1. World Energy Council. 2014 World Energy Issues Monitor. 2014. Available online: https:// www.worldenergy.org/wp-content/uploads/2014/01/World-Energy-Issues-Monitor-2014.pdf (accessed on 8 August 2016).

2. European Commission. The Future Role and Challenges of Energy Storage. 2012. Available online: https://ec.europa.eu/energy/sites/ener/files/energy_storage.pdf (accessed on 8 August 2016).

3. U.S. Department of Energy. Grid Energy Storage. December 2013. Available online: http://energy.gov/ sites/prod/files/2013/12/f5/GridEnergyStorageDecember2013.pdf (accessed on 8 August 2016).

4. Arnold, S.M.; Salee, A.F.; Al-Zoubi, N.R. Deformation and Life Analysis of Composite Flywheel Disk and Multi-Disk Systems; National Aeronautics and Space Administration, Glenn Research Center: Cleveland, OH, USA, 2001.

5. Bolund, B.; Bernhoff, H.; Leijon, M. Flywheel energy and power storage systems. Renew. Sustain. Energy Rev. 2007, 11, 235-258. [CrossRef]

6. Hansen, G.R.; O'Kain, U. An Assessment of Flywheel High Power Energy Storage Technology for Hybrid Vehicles. Available online: http://www.compositesworld.com/cdn/cms/ORNLFlywheelAssessmentfor HybridVehicles2011.pdf (accessed on 8 August 2016).

7. Fiske, O.J.; Ricci, M.R. Third generation flywheels for high power electricity storage. In Proceedings of the 19th International Conference on Magnetically Levitated Systems and Linear Drives, Dresden, Germany, 13-15 September 2006.

8. Schweitzer, G.; Eric, H. Maslen. Magnetic Bearings; Springer: Berlin/Heidelberg, Germany, 2009.

9. Abrahamsson, J.; Hedlund, M.; Kamf, T.; Bernhoff, H. High-Speed Kinetic Energy Buffer: Optimization of Composite of Composite Shell and Magnetic Bearings. IEEE Trans. Ind. Electron. 2014, 61, 3012-3021. [CrossRef]

10. Han, B.; Xu, Q.; Yuan, Q. Multiobjective Optimization of a Combined Radial-Axial Magnetic Bearing for Magnetically Suspended Compressor. IEEE Trans. Ind. Electron. 2016, 63, 2284-2293.

11. Le, Y.; Sun, J.; Han, B. Modeling and Design of 3-DOF Magnetic Bearing for High-Speed Motor Including Eddy-Current Effects and Leakage Effects. IEEE Trans. Ind. Electron. 2016, 63, 3656-3665. [CrossRef]

12. Cheng, S.; Day, S.W. Design and Control of Hybrid Magnetic Bearings for Maglev Axial Flow Blood Pump. In Proceedings of the 2010 IEEE/ ASME International Conference on Advanced Intelligent Mechatronics, Montreal, QC, Canada, 6-9 July 2010; pp. 187-192.

13. Zhang, W.; Hu, Y. A Prototype of Flywheel Energy Storage System Suspended by Active Magnetic Bearings with PID controller. In Proceedings of the Asia-Pacific power and Energy Engineering Conference (APPEEC 2009), Wuhan, China, 27-31 March 2009; pp. 1-4.

14. Schuhmann, T.; Hofmann, W.; Werner, R. Improving Operational Performance of Active Magnetic Bearings Using Kalman Filter and State Feedback Control. IEEE Trans. Ind. Electron. 2012, 59, 821-829. [CrossRef]

15. Rundell, A.E.; Drakunov, S.V.; DeCarlo, R.A. A Sliding Mode Observer and Controller for Stabilization of Rotatinal Motion of a Vertical Shaft Magnetic Bearing. IEEE Trans. Control Syst. Technol. 1996, 4, 598-608. [CrossRef]

16. Jiang, K.; Zhu, C.; Chen, L. Unbalance Compensation by Recursive Seeking Unbalance Mass Position in Active Magnetic Bearing-Rotor System. IEEE Trans. Ind. Electron. 2015, 62, 5655-5664. 
17. Halbach, K. Design of permanent multipole magnets with oriented rare earth cobalt material. Nuclear Instrum. Methods 1980, 169, 1-10. [CrossRef]

18. Jang, S.M.; Lee, S.H.; Cho, H.W.; Cho, S.K. Analysis of unbalanced force for high-speed slotless permanent magnetic machine with Halbach array. IEEE Trans. Magn. 2003, 39, 3265-3267. [CrossRef]

19. Sun, J.; Ren, Y.; Fang, J. Passive axial magnetic bearing with Halbach magnetized array in magnetically suspended control moment gyro application. J. Magn. Magn. Mater. 2011, 323, 2103-2107.

20. Choi, Y.M.; Gweon, D.G. A high-precision dual-servo stage using halbach linear active magnetic bearings. IEEE/ASME Trans. Mechatron. 2011, 16, 925-931. [CrossRef]

21. Gruber, W.; Huber, M.; Jungmayr, G.; Amrhein, W. Passive tilt bearings with zero translational Stiffness composed of two permanent magnet rings. In Proceedings of the Twelfth International Symposium on Magnetic Bearings, Wuhan, China, 23-25 August 2010; pp. 21-26.

22. Tian, L.L.; Ai, X.P.; Tian, Y.Q. Analytical model of magnetic force for axial stack permanent-magnet bearings. IEEE Trans. Magn. 2012, 48, 2592-2599. [CrossRef]

23. Bachovchin, K.D.; Hoburg, J.F.; Post, R.F. Magnetic fields and forces in permanent magnet levitated bearings. IEEE Trans. Magn. 2012, 48, 2112-2120. [CrossRef]

24. Bachovchin, K.D.; Hoburg, J.F.; Post, R.F. Stable levitation of a passive magnetic bearing. IEEE Trans. Magn. 2013, 49, 609-616. [CrossRef]

25. Wang, N.; Wang, D.; Chen, K.; Wu, H. Research on analytical model and design formulas of permanent magnetic bearings based on Halbach array with arbitrary segmented magnetized angle. J. Magn. Magn. Mater. 2016, 401, 257-264. [CrossRef]

26. Khalil, H.K. Nonlinear Systems, 2nd ed.; Prentice-Hall: Upper Saddle River, NJ, USA, 1996.

(C) 2016 by the authors; licensee MDPI, Basel, Switzerland. This article is an open access article distributed under the terms and conditions of the Creative Commons Attribution (CC-BY) license (http:/ / creativecommons.org/licenses/by/4.0/). 